\title{
Achón Rodríguez Olga, Importando miseria
}

Thibaud de Fortescu

\section{(2) OpenEdition}

\section{Journals}

Édition électronique

URL : https://journals.openedition.org/remi/6968

DOl : $10.4000 /$ remi.6968

ISSN : $1777-5418$

Éditeur

Université de Poitiers

Édition imprimée

Date de publication : 1 décembre 2014

Pagination : 231-232

ISBN : 979-10-90426-23-8

ISSN : 0765-0752

Référence électronique

Thibaud de Fortescu, «Achón Rodríguez Olga, Importando miseria », Revue européenne des migrations internationales [En ligne], vol. 30 - n³ et 4 | 2014, mis en ligne le 01 décembre 2014, consulté le 14 avril 2022. URL : http://journals.openedition.org/remi/6968; DOI : https://doi.org/10.4000/remi.6968 
Achón Rodríguez, Olga

Importando miseria. - Madrid : Catarata, 2011. -229 p.

ISBN : 978-84-8319-621-2

Le livre d'Olga Achón est issu de sa thèse de doctorat soutenue en 2011 à I'Université de Barcelone et portant sur le recrutement en origine des travailleurs immigrés mis en place par le syndicat Unió de Paguesos (UP) dans la province de Lérida (Catalogne). L'ouvrage, divisé en trois chapitres, démontre de quelle manière un système est mis en place avec l'aval de l'État afin d'importer de la main-d'œuvre et d'exercer un contrôle strict sur les travailleurs une fois sur place, tout cela dans un contexte général d'organisation des migrations internationales. L'auteure, qui a travaillé pour le syndicat avant de commencer sa thèse, s'appuie sur une enquête de terrain étayée par de nombreux témoignages, que ce soit du côté des immigrés, du syndicat ou des responsables publics.

Le premier chapitre aborde les origines du système et notamment l'ensemble des dispositifs législatifs qui en ont permis l'émergence. L'auteure rappelle que la première initiative de recrutement en origine (système permettant à un organisme de faire venir des travailleurs sur la base d'un contrat précis tout en ayant l'assurance qu'ils repartent une fois le contrat terminé) date de 1999, avec le recrutement de trente-cinq travailleurs colombiens. C'est cette même année que fut signé le "Protocole additionnel à l'accord de collaboration pour l'organisation des migrations intérieures dans les diverses campagnes agricoles d'emplois temporaires " par le ministère du Travail et des Affaires sociales et l'ensemble des organisations agricoles. Ce chapitre présente un grand intérêt, car il nous rappelle que les travailleurs font face à de réelles difficultés pour se libérer des conditions de travail parfois précaires. Le statut juridique accepté, de fait, en signant leur contrat, ne leur permet pas d'exercer certains droits et il est, par exemple, impossible d'abandonner le travail ou de choisir son employeur. UP impose des conditions qui influent sur la vie privée des travailleurs, comme l'obligation de résider dans le logement attribué, d'abandonner son emploi ou de retourner dans son pays à la fin du séjour ; conditions toutes incluses dans le contrat. L'auteure rappelle l'ambiguïté du rôle du syndicat qui est à la fois syndicat et fonction publique. En tant que syndicat il n'a donc pas de pouvoir disciplinaire spécifique, puisque son rôle d'administration lui permet déjà de faire peser une menace d'expulsion. Olga Achón rappelle toutefois que l'expulsion est assez peu appliquée, mais qu'à l'inverse les déplacements de travailleurs d'un site à l'autre sont fréquents, de façon à trouver une alternative aux problèmes et surtout, à ne pas perdre d'argent. En effet, la fugue ou l'abandon de poste d'un travailleur mettrait le système en échec.

Le deuxième chapitre - le plus court de I'ouvrage - consiste essentiellement en un retour historique, à la fois sur les lois espagnoles et européennes, avec une attention particulière portée au système prussien qui concernait des travailleurs polonais. Au fur et à mesure de l'augmentation du phénomène migratoire, l'État a consenti à la présence de travailleurs étrangers en situation irrégulière, car ils représentent la main-d'œuvre idéale, car rentable et docile, au sein d'un marché du travail segmenté. Cependant, la figure de l'étranger irrégulier pose problème, notamment dans le cadre européen, car elle est perçue comme facteur de désordre et créatrice de peurs. 
C'est pourquoi l'État a mis en place des politiques publiques visant, entre autres, à éradiquer cette figure de l'espace public. L'auteure s'empresse donc d'effectuer un retour sur les législations passées et en cours. Elle démontre que l'exploitation des travailleurs temporaires ne constitue pas en tant que telle une nouveauté et que, par conséquent, le système en cours en Catalogne peut aussi s'apparenter à un système archaïque.

Le troisième et dernier chapitre est centré sur la question du logement, élément clé de l'analyse, car c'est par ce biais que UP exerce le plus fortement son pouvoir et met en place un mode de contrôle du travailleur. La figure du responsable de logement est extrêmement importante, car ces personnes contribuent à imposer des normes et des fonctionnements contraignants pour le travailleur. Les responsables des logements sont souvent des femmes, diplômées en " éducation sociale " ou " travail social ", que l'auteure a tendance à décrire comme faisant preuve de bonne volonté, mais étant contraintes à se plier aux exigences du syndicat. $\mathrm{Ce}$ dernier impose des règlements intérieurs très stricts. En effet, le contrôle récurrent de I'hygiène du logement et du travailleur, I'interdiction de l'alcool, l'impossibilité de dormir une nuit à l'extérieur (car cela nuit au travail du lendemain) ou la réglementation des visites contribuent à instaurer un climat de méfiance vis-à-vis des immigrés. L'auteure apporte une attention particulière à décrire les différences entre les deux types de logements existants : privés et publics. Au sein de la première catégorie, il existe deux sous-groupes : ceux appartenant aux petits agriculteurs et ceux des entreprises agricoles. Les premiers sont plus petits et concernent majoritairement des travailleurs étrangers locaux ou vivant dans d'autres régions d'Espagne. Les seconds, ayant des responsables employés par le syndicat, concernent les travailleurs recrutés par le biais de contrats en origine. Les logements publics sont propriétés des mairies ou d'autres administrations. Ils font office de réserve de main-d'œuvre, afin de pallier le manque de travailleurs. C'est ici que le système se déploie avec la plus forte intensité et c'est le type de logement qui convient le mieux à la mise en place d'une circulation constante et systématisée des travailleurs.

Par sa démonstration, Olga Achón met en lumière l'existence d'un système créateur d'inégalités légitimé par l'État espagnol et I'Union européenne. Tout au long de son travail, l'auteure ne cache pas son orientation et sa critique vis-à-vis du système mis en place par le syndicat et l'État, ce qui tend parfois à rendre le propos un peu moins convaincant (lorsque les travailleurs sont, par exemple, comparés aux esclaves vivant à Rome à l'époque de Caton). L'ouvrage s'avère toutefois être extrêmement riche et ouvre des perspectives de travail intéressantes dans plusieurs disciplines, notamment en géographie (sur la ségrégation sociospatiale des immigrés) et en psychologie sociale (sur le lien entre les immigrés et les travailleurs sociaux). Les nombreuses références historiques et juridiques contribuent également à replacer ce travail dans un cadre plus large et accentuent la pertinence du propos. Depuis la parution du livre, il conviendrait de connaitre l'impact de la crise économique sur ce système.

Thibaud de Fortescu

Centre de Recherches et d'Analyses

Géopolitiques (Paris 8)

Université de Strasbourg

Adjemian, Boris

La fanfare du Négus : les Arméniens en

Éthiopie (XIXe-XXe siècles). - Paris : Éditions EHESS, 2013. $-350 \mathrm{p}$.

ISBN : 978-2- 7132-2415-7

Cette étude, issue d'un doctorat en histoire contemporaine réalisé sous la direction de Gérard Noiriel et d'Alessandro Triulzi, portant sur la migration d'Arméniens en Éthiopie, frappe par sa grande origi- 
nalité. Dans un remarquable style d'écriture, son auteur présente I'histoire d'une migration originale, qui a concerné au plus fort un petit millier de personnes, mais qui est révélatrice des processus de construction de l'État-nation éthiopien depuis la fin du XIXe siècle. Résolument innovante, elle observe les formes de sédentarisation d'une diaspora dans un pays alors hostile à toute présence étrangère.

L'histoire de cette fanfare, composée d'orphelins du génocide, et invités en 1924 à rejoindre Addis-Abeba par le futur empereur afin de composer sa garde musicale, n'est qu'un prétexte pour une étude sur le temps long de cette migration. En éclairant les relations entre les églises arméniennes et du pays hôte, comme en travaillant sur les routes commerciales du XIXe siècle entre l'Arménie et la Corne de l'Afrique, l'auteur montre comment le champ migratoire s'est structuré pour se développer après les massacres de 1915-1916. Là où un travail de " facture classique " se serait contenté d'une approche par aire géographique, ou d'une étude en termes de " communauty studies ", dans laquelle on trouverait une histoire convenue de l'exil, I'auteur mobilise les différents concepts de diaspora, forgés par les disciplines sociologiques et géographiques, pour comprendre l'appropriation des lieux en migration. C'est là un changement de paradigme intéressant : le regard est porté non sur les liens transnationaux des individus en diaspora, mais sur la construction des sédentarités dans les nouveaux espaces d'habitation. En parallèle, l'approche résolument sociohistorique met au jour les dominations politiques qui se sont exercées sur les individus, et les marges de manœuvre dont ils disposaient pour organiser leur implantation et leur pérennisation dans le pays. Cela éclaire non seulement l'histoire de l'autonomie des migrants, mais montre aussi, dans le même temps, comment l'État éthiopien, de façon tout à fait originale, a intégré une population " apatride " pour mieux affirmer sa résistance aux tenta- tives coloniales européennes. C'est-là un modèle tout à fait inédit, à contrecourant des processus politiques d'exclusion des États-nations européens, et qui mérite l'attention.

Ce travail est aussi une belle invite à réfléchir les méthodologies d'enquête. Les archives officielles sur cette population étant particulièrement lacunaires, Boris Adjémian a su recueillir de multiples sources. Comme l'a préconisé Marc Bloch, il a tout d'abord arpenté les lieux de son étude. En quête des traces laissées par les populations, il observe avec minutie AddisAbeba, dont les façades des bâtiments et les enseignes d'anciens magasins. II se rend dans la cathédrale, les cimetières, les écoles et des thermes pour aussi comprendre l'espace social de cette migration. De même, le chercheur a pratiqué une écoute active auprès des descendants d'Arméniens ; et a obtenu d'eux la possibilité de consulter leurs archives, dont de fascinants albums photographiques, savamment construits par leurs propriétaires pour mettre en scène les événements communautaires marquants (et dont quelques belles photographies sont reproduites dans l'ouvrage). Mais ici encore, I'originalité de son approche ne réside certainement pas dans une enquête qui circonscrirait une communauté et recueillerait toutes les anecdotes possibles auprès des détenteurs et producteurs de mémoires. Bien au contraire Boris Adjémian bâtit une réelle réflexion sur les modalités de construction d'une " autobiographie collective " portée par les responsables communautaires. En considérant leurs propos sur la proximité des Arméniens avec la cour impériale, il reconstitue les enjeux discursifs qui, dans le temps passé comme dans le moment présent, ont servi et servent encore à appuyer la légitimité des Arméniens à résider en Éthiopie. Cette " mémoire d'hôte " mérite l'attention. Boris Adjémian, a su ainsi saisir la subtilité de ces constructions et montre très clairement comment dans le temps long communautaire, les discours ont été 
gommés de toutes aspérités tandis que les actes des Arméniens, notamment dans leur recherche de protection consulaire, pouvaient contredire cette posture officielle. II montre aussi que contrairement aux Arméniens d'Europe, il n'y a pas eu de politisation de la mémoire du génocide durant la décennie 1970. Bien au contraire, les efforts communautaires ont plutôt consisté, au moment de la révolution de 1974, à rappeler la très ancienne allégeance du groupe à son nouveau pays.

Aussi, au-delà de I'histoire passionnante qu'il propose, l'ouvrage de Boris Adjémian se conçoit comme un manuel d'enquête en science sociale, dans lequel il est aisé de puiser des pistes de recherche. En ce sens, cet ouvrage doit occuper une place inédite dans les préoccupations intellectuelles de ceux intéressés à l'étude des faits migratoires.

Yann Scioldo-Zürcher Historien, Chargé de recherche CNRS Centre de recherche français à Jérusalem

\author{
Cossée, Claire (dir.) \\ Miranda, Adelina (dir.) \\ Ouali, Nouria (dir.) \\ Séhili, Djaouida (dir.) \\ Le genre au cœur des migrations. - Paris : \\ Éditions Petra, 2012. - 336 p. \\ ISBN : 979-2-84743-056-3
}

L'ouvrage collectif Le genre au cœur des migrations inaugure la collection " intersectionS " des éditions Petra. Il contribue de la sorte à l'ouverture d'un espace d'écriture sur la construction de rapports de domination imbriqués. L'événement mérite d'être souligné à deux titres. En premier lieu, cet ouvrage n'a rien de classique. II participe à I'institutionnalisation récente d'un champ de recherche en France, celui de la perspective du genre appliquée à l'étude des migrations. Pionnière de cette approche dans I'hexagone, Mirjana Morokvasic introduit l'ouvrage en retraçant les étapes successives par lesquelles les études sur l'immigration et sur les femmes et les rapports sociaux de sexe se sont enfin rencontrés dans les années 1970 et 1980 . Bien que nous observions aujourd'hui un nombre conséquent de travaux analysant ce que les processus migratoires, les expériences des migrant-e-s et les impacts politiques des migrations ont de genré, ceux-ci s'avèrent éparpillés dans l'espace universitaire français. Un tel ouvrage contribue à rassembler des questionnements et des innovations méthodologiques que nourrit cette perspective de recherche. En second lieu, il semble opportun de remarquer que ce sont les objets du champ des migrations qui conduisent à introduire la lecture articulée des rapports sociaux de genre, de classe, de " race " et de sexualité à l'étude du pouvoir et des dominations en France.

Ce travail d'écriture collectif est issu d'un colloque pluridisciplinaire organisé à Paris VIII par le groupe "Genre et migrations " du laboratoire GTM, devenu aujourd'hui le CRESPPA (CNRS Paris VIIIParis X), en partenariat avec MinorityMedia (Université de Poitiers) et le GEM (Groupe d'Études et de Recherches Genre et Migration, Université Libre de Bruxelles). II se lit en quatre parties qui proposent chacune un outil ou un objet de recherche présenté par l'une des co-auteures avant d'introduire l'ensemble des contributions. Sont alors traités les échelles migratoires, I'articulation des rapports sociaux, les accès contrastés à l'emploi et les féminismes et mobilisations.

Dans la première partie, Adelina Miranda présente l'articulation des échelles migratoires comme un outil théorique et méthodologique impérieux pour saisir l'ordre genré qui organise les mobilités selon divers niveaux spatio-temporels. Elle distingue trois échelles potentielles d'analyse afin d'appréhender le genre des champs migratoires : celle qui croise les niveaux individuel et familial, celle qui emboîte les contextes local, régional et global, et celle qui articule les acteurs 
sociaux des migrations qui agissent à différentes échelles du pouvoir. Par la notion de " carrière migratoire ", Laurence RoulleauBerger désigne à la fois les inégalités multisituées subies par les femmes migrantes à chaque étape de leur migration et leur participation active aux nouvelles formes circulatoires et multiscalaires, locales et globales, des marchés du travail. En variant les échelles d'observation en Amérique latine, Sara María Lara Flores démontre que l'assignation des femmes sur le marché du travail précaire est une constante migratoire, que ce soit lors des déplacements internes, intra-régionaux ou transnationaux. Elle articule les facteurs économiques avec les motivations familiales et individuelles pour comprendre la logique sexuée qui régit la sélectivité des migrant-e-s. Eleonor Kofman poursuit l'effort de décentrement des perspectives trop souvent orientées vers les migrations du Sud vers le Nord, concernant en particulier les migrations des femmes dans les métiers du care. Elle développe en suivant I'outil du care diamond pour appréhender les différents régimes de care reliés par les migrations et pour prendre en compte les divers acteurs de ces chaînes globales du care, sans en omettre l'État.

Dans une deuxième partie, Nouria Ouali retrace une sociographie des approches intersectionnelles qui aident à accomplir une démarche de recherche visant à saisir les multiples aspects de la domination inhérente aux phénomènes migratoires. En même temps qu'elles considèrent que I'articulation des rapports sociaux de classe, de " race " et de sexualité est essentielle à l'étude des rapports de genre, les auteures des textes réunis dans cette partie rendent également compte des difficultés méthodologiques à manipuler l'outil intersectionnel. Étudiant les migrations antillaises en métropole, Stéphanie Condon insiste sur le rôle que joue l'histoire coloniale sur la division sexuée des emplois en migration et sur les barrières aux mobilités professionnelles des migrant-e-s. Jules Falquet éclaire quant à elle, par le biais de l'étude de l'hyper-mobilité et de la faiblesse des droits reconnus aux lesbiennes, la contrainte à I'hétéro-circulation et la pression à la conjugalité à laquelle sont soumises toutes les femmes migrantes. Elle met également en saillance par ce cas le caractère éminemment transgressif de la migration pour les femmes, notamment lorsqu'elles partent seules. Par l'entremise d'une histoire de l'émigration et de l'immigration des femmes en Italie, Adelina Miranda insiste sur la " racialisation " des femmes migrantes, ainsi que sur les changements économiques et culturels qu'elles introduisent dans les rapports sociaux de sexe du monde domestique. Emmanuelle Lada prescrit par sa contribution d'analyser les discriminations ethniques au travail en France dans une perspective de genre.

Djaouida Séhili oriente la troisième partie vers un lieu privilégié d'observation de l'intrication des rapports sociaux, celui du travail et du marché de l'emploi. Enrichissant la sociologie du travail d'une sociologie des migrations et du genre, les contributions interrogent les phénomènes d'assignation et d'exclusion des femmes migrantes et des descendantes d'immigrée-s de certains secteurs professionnels. Usant de la comparaison, Laura Oso et Christine Catarino appréhendent la féminisation des migrations et l'insertion des femmes migrantes dans certains secteurs d'emploi en Espagne et au Portugal en observant leur corrélation avec les fluctuations de la demande en main-d'œuvre masculine dans ces pays. En s'intéressant aux descendantes d'immigré-e-s en Belgique, Nouria Ouali met en évidence la façon dont les projets migratoires familiaux et la stigmatisation des filles d'immigrée-s jouent sur leur rapport aux ambitions professionnelles, qu'elle oppose à celui des filles de parents autochtones.

Toujours animée par une perspective intersectionnelle, la dernière partie coordonnée par Claire Cossée rend explicite les formes de marginalisation de l'expérience des minoritaires des mobilisations 
féministes et du militantisme auprès de populations perçues comme assistées, par exemple les personnes sans-papiers. Proposant des outils d'observation de l'imbrication des rapports sociaux au sein des mobilisations, la démarche scientifique adoptée par les différentes auteures veut donner voix à des discours constamment occultés et offrir ainsi un point de vue alternatif aux lectures essentialistes des revendications de groupes sociaux variés. Nasima Moujoud complexifie ainsi le rapport des femmes sans-papiers aux espaces militants et au féminisme en fonction de leur classe sociale d'origine et de leur niveau de qualification. De la même manière qu'elle expose la façon dont les mobilisations féministes reproduisent les frontières de classe et de " race " entre femmes dans la répartition des tâches militantes, Gily Coene et Ilke Adam pointent la marginalisation du point de vue des femmes issues de groupes minoritaires des débats sur le voile et du féminisme en Belgique. La traduction d'un article d'Alexandra Oprea qui termine cet ouvrage permet de démontrer plus encore, à travers le cas des revendications émancipatrices des femmes roms, l'exclusion des discours sur les inégalités de genre des programmes et des luttes pour ou par certaines catégories de population.

Les contributions recueillies au sein de cet ouvrage parviennent à convaincre que le genre est une grille de lecture fondamentale des processus migratoires. Réciproquement, elles font apparaître que le champ des migrations tend à guider la perspective du genre vers des problématiques qui s'enrichissent des expériences des femmes migrantes à des positions sociales différentes dans les rapports de domination. Proposant des outils d'analyse, comparant des groupes de population, variant les échelles d'observation et les questionnements de recherche, l'ouvrage apporte des éléments de compréhension de la soudaine mise en visibilité des femmes dans les migrations par les médias, tandis qu'il lève le voile sur un ensemble de phénomènes migratoires qui restent encore peu connus.

Colette Le Petitcorps Doctorante en sociologie MIGRINTER/Université de Poitiers

Fassin, Didier (dir.)

Les nouvelles frontières de la société française. - nouvelle édition. - Paris : Éditions La Découverte, 2012. - 599 p. ISBN : 978-2-7071-7456-6

Didier Fassin a réuni une équipe multidisciplinaire qui a effectué une série d'enquêtes établissant l'impressionnant catalogue des frontières juridiques, psychologiques, symboliques par lesquelles la société française se trouve fractionnée en cellules inégales.

Les auteurs soulignent d'abord l'importance des limites territoriales qui séparent traditionnellement les citoyens et les étrangers. Une autre frontière est instituée par la connaissance de la langue française, plus ou moins maîtrisée, considérée depuis 1890 comme un critère d'assimilation, garantissant l'adhésion aux valeurs républicaines, facilitant ou non la naturalisation. Cette dernière ne permet pas toujours une acceptation du nouveau citoyen dans la société, car le nom et l'apparence peuvent demeurer comme des rappels discriminants de l'origine étrangère. De toute manière, la différence, de quelque nature qu'elle soit, peut constituer un handicap et maintenir une frontière plus ou moins contraignante. La nationalité a peu de poids en la matière : même si la biologie prouve l'unité du genre humain et si la notion de " race " apparaît comme une construction sociale, le regard et le comportement de certains individus, la loi parfois, érigent des barrières. Le livre donne bien d'autres exemples de séparations, entre les individus jouissant de leur liberté de mouvement et ceux qui sont enfermés dans des centres de rétention, entre ceux qui subissent les rigueurs de la 
loi sur le sol français et ceux qui, sous la pression du pays dit d'accueil, sont retenus dans leur pays d'origine, entre ceux qui vivent entourés de leur famille et ceux qui en sont séparés par les procédures d'un regroupement familial de plus en plus sévère. Les étrangers finissent parfois par intérioriser la frontière sous l'effet de la peur qu'ils éprouvent, ce qui dans certains cas peut engendrer des troubles mentaux.

Les auteurs réservent une place aux Français qui, d'une manière ou d'une autre, luttent contre les discriminations et font acte de solidarité. Ainsi les militants humanitaires, les membres du Réseau éducation sans frontière, les associations chargées par l'État de gérer les procédures d'asile, ceux des policiers qui essaient de légitimer leur action ou d'adoucir les rigueurs de la loi. Mais, dans l'ensemble, le bilan apparaît très sombre. Le livre brosse le portrait d'une France cadenassée, hérissée de règlements coercitifs, marquée par des comportements quotidiens xénophobes ou racistes. Les auteurs ne croient que les responsables politiques et administratifs qui gèrent sévèrement l'immigration répondent toujours aux exigences d'une opinion publique inquiète : la " xénophobie de gouvernement " utiliserait les prétendues pressions de l'opinion pour justifier des choix anti-étrangers (p. 178). La présence de représentants de la " diversité " sur les listes électorales constituerait " un cache-misère, une opération éphémère de diversion politique " (p. 424). L'adoption d'un comportement humain et progressiste par la droite et le monde économique serait un trompe-l'œil, car " le respect des droits fondamentaux d'une part et les performances économiques d'autre part " sont difficilement conciliables (p. 264). Du fait que l'inégalité sociale correspond souvent aux inégalités ethniques, " l'État ne mène plus la guerre à la pauvreté mais aux pauvres " (p. 98). L'Europe est devenue un "Empire du rejet " qui " correspond à un état de domination des pays du Nord sur ceux du Sud dont la vie politique se transforme en fonction des finalités de ceux du Nord " (p. 193). « Les majoritaires qui détiennent le pouvoir tant économique que politique n'ont aucun intérêt à faire émerger les questions de ce genre, les questions raciales, ni aucune autre question liée aux discriminations $"$ (p. 288).

Certains auteurs nuancent le constat accablant auquel ils parviennent en notant que les échantillons d'individus, parfois inférieurs à dix, sur lesquels ils ont travaillé sont modestes et constituent plus des études de cas que des bilans généralisables à toute la société. Cependant les plus nombreux n'hésitent pas à énoncer des conclusions globales. Chaque lecteur souscrira ou non à ces conclusions en fonction de sa propre expérience ou de sa philosophie. On ne peut certes nier que le national appartient traditionnellement à une catégorie privilégiée par rapport à l'étranger. L'éventuelle extension de certains privilèges aux non-Français ou la redéfinition d'une citoyenneté qui ne serait plus liée au territoire et serait comme dit Étienne Balibar « une communauté de destin " (Les Frontières de la démocratie, La Découverte, 1992) appellent des réponses politiques. Les chercheurs, de manière plus ou moins convaincante, contribuent à mettre en lumière les tensions qui parcourent la société et proposent de celle-ci un tableau qui stimule la discussion.

Ralph Schor

Département d'histoire Université de Nice-Sophia-Antipolis

Spire, Amandine

L'étranger et la ville en Afrique de l'Ouest. Lomé au regard d'Accra. - Paris : Karthala, 2011. - 378 p. (Collection Hommes et sociétés)

ISBN : 978-2-8111-0439-9

D'aucuns apprécieront cette nouvelle publication, de très bonne facture, sur les questions urbaines en Afrique de 
I'Ouest signée aux éditions Karthala. Cet ouvrage qui consacre une nouvelle thèse de géographie entreprise sur une ville africaine (Lomé) complète les travaux de doctorat récemment publiés dans la même collection (Mansour Tall sur Dakar, Armelle Choplin sur Nouakchott). Dans la sous-région, les grandes villes littorales ont longtemps été étudiées en relation avec les migrations internes qui ont, pour un temps, largement animé leur peuplement et ainsi contribué à leur fabrique. Ici, par le jeu d'un changement d'échelle, l'auteure s'emploie à décrypter le produit des interactions entre la présence étrangère à Lomé et les dynamiques spatiales récentes observées dans la capitale togolaise. C'est donc sous I'angle des migrations et des circulations internationales que sont questionnées les transformations urbaines locales et l'émergence de pratiques " hybrides " participant aux recompositions de la ville. La démarche est à la fois originale et ambitieuse. En y transposant sa problématique dans les mêmes termes, le cas de la ville d'Accra (Ghana) est mobilisé, à bon escient, par Amandine Spire qui en fait un " miroir " utile pour enrichir son point de vue porté sur Lomé : le choix d'un regard croisé, assumé et défendu par la chercheure, raffine ses questionnements, tempère l'interprétation les résultats de son enquête ethnographique, appelle une prise de recul nécessaire sur ses conclusions. L'approche comparative prend alors tout son sens parce qu'elle sert avant tout à se distancer de "l'objet " et à souligner les spécificités locales des processus appréhendés.

Posant d'emblée l'hypothèse de la dispersion des étrangers à l'échelle de la ville et donc de l'absence de quartiers ethniques, le positionnement théorique consiste d'abord à discuter certaines thèses émanant de la tradition sociologique de Chicago et du paradigme de l'exode rural, pour finalement mieux souligner la singularité des dynamiques urbaines d'Afrique de l'Ouest entraînées par la présence de migrants étrangers (chapitre 1). Ces acteurs émergent sur la scène urbaine loméenne derrière la figure de "l'aventurier " porteur de nouvelles valeurs citadines (chapitre 2). L'origine de ces étrangers est analysée à travers l'examen des trajectoires migratoires en insistant bien sur les temporalités dans les parcours et les modalités de l'ancrage, en particulier au moment crucial de l'arrivée en ville (chapitre 3). Une attention particulière est portée à la localisation des gares routières qui constituent autant de "portes d'entrée " sélectives des étrangers parvenant à Lomé. Les belles pages qui suivent sur la frontière et les pratiques " transnationales " (chapitres 4 et 5) déroutent un peu le lecteur avant son immersion plus attendue au " cœur " de la ville. Trois études de quartiers et des analyses à l'échelle micro permettent ainsi d'affiner la connaissance des processus locaux œuvrant à la production d'une citadinité étrangère dans des registres plus ou moins visibles, en fonction des échelles et des contextes considérés (chapitres 6, 7 et 8).

L'histoire des zongos, formes spatiales singulières des systèmes urbains de la partie orientale de l'Afrique de I'Ouest, rythmés par un double mouvement " mobilité sur de grandes distances d'un certain nombre de ses habitants venants d'un ailleurs, mais mobilité également du quartier à l'échelle de la ville par des déguerpissements successifs " constitue un point remarquable de l'analyse des interactions entre la dynamique urbaine et l'évolution, la reconstruction des identités étrangères. Autre forme urbaine originale dans le paysage loméen, le quartier de Katanga apparenté à un "bidonville ", sinon à un "village " essentiellement peuplé de migrants ghanéens, illustre à son tour la complexité et la diversité des processus de création urbaine produits par les identités étrangères dans un rapport ambigu avec les autorités locales.

Enfin, face à l'essaimage spatial des étrangers constaté ailleurs dans la ville, le dernier chapitre est consacré aux 
modalités d'invention de la ville par les étrangers à l'échelle micro et dans le temps du quotidien. Cette « micro-géographie " constitue certainement la partie la plus aboutie et aussi la plus explicite de la démonstration d'Amandine Spire, ce que ne manque pas de relever Philippe Gervais-Lambony, son Directeur de thèse, dans la belle postface qu'il lui dédie en fin d'ouvrage. Deux types de lieux sont choisis : la cour (espace résidentiel partagé par plusieurs ménages et faisant cohabiter Loméens et étrangers) et les cafétérias ou kiosques $^{1}$ à Accra. C'est dans la maison, " métaphore du moi " territorialisé avec sa dimension affichée et familière (Di Méo, 1996), et dans ses modes de cohabitation que l'on saisit au mieux l'empreinte invisible de la circulation résidentielle qui anime de manière singulière le parc locatif loméen. Et, c'est par cette forme de bistrot de rue, les cafétérias, que prend le mieux forme l'empreinte étrangère sur la ville : un style de commerce produit " par " et " dans " la migration des Guinéens à l'étranger, sans que ces derniers ne reproduisent une pratique du pays d'origine.

Finalement, en termes d'impacts sur la ville, I'hypothèse qui est validée est bien que l'ancrage des étrangers en ville provoque la prise de possession de certains lieux, vecteur d'une appartenance à l'ailleurs redéfinie dans des interactions locales. Autrement dit, le maintien d'identités étrangères à la ville ne repose pas sur la réplique d'identités qui apparaissent ailleurs ou dans d'autres temps, mais semble bien le produit d'une différenciation et d'une création identitaire " dans " et " de " la ville. En définitive, l'étranger est un acteur de la citadinité à part entière, dans la mesure où il se sent suffisamment appartenir à la ville pour être en mesure de la transformer.

1 Lieux de consommation et de socialisation presque toujours ouverts sur la rue, proche du fonctionnement des bars, disposant en plus d'un service de restauration légère.
Amandine Spire nous livre un texte d'une lecture agréable et vivante agrémenté par un sens aiguisé de la description. L'ouvrage est documenté par de nombreuses cartes, photographies et encadrés illustrant, avec sensibilité et justesse, I'histoire des figures étrangères rencontrées et les "scènes urbaines " où sont observées leurs pratiques. L'auteure nous conduit habilement dans sa traversée de Lomé et ses étapes à Accra, et si I'on se perd parfois un peu dans la progression générale de l'ouvrage, c'est qu'Amandine Spire ne dissimule pas la complexité de sa recherche qui fait appel à de nombreuses dimensions et nécessite un recours incessant à des changements d'échelle dans le temps et dans l'espace : de l'agglomération urbaine à l'habitat de cour, du temps long des parcours migratoires à l'échelle du quotidien.

On pourra s'étonner que l'approche développée n'ait que trop timidement été nourrie par différentes avancées théoriques et méthodologiques récentes sur les recompositions urbaines des villes du Sud; en particulier dans le domaine des interactions entre mobilités et dynamiques intra-urbaines et dans le domaine des transports et des circulations internationales en Afrique de l'Ouest. Ce détour aurait sans doute permis au lecteur de mieux situer l'enjeu des migrations internationales dans la dynamique urbaine d'ensemble et en lien avec d'autres formes tout aussi importantes de la mobilité spatiale. L'écueil à éviter serait alors de tomber dans une sur-exposition ou survalorisation du rôle de l'étranger ou du migrant international dans la production et I'organisation urbaine locale.

David Lessault MIGRINTER CNRS/Université de Poitiers 
Wihtol de Wenden, Catherine (Dir.)

La Turquie au carrefour des turbulences migratoires : hier et aujourd'hui. - Paris, CNRS Éditions, 2012. - 263 p. (Anatoli. De l'Adriatique à la Caspienne. Territoires, politique, sociétés ; $n^{\circ} 3$ ) ISBN : 978-2-271-07539-0

Anatoli est la nouvelle dénomination d'une revue plus ancienne créée par Semih Vaner, Directeur de Recherche au CNRS et membre du CERI (qui nous a quitté prématurément en 2008 des suites d'une longue maladie), les CEMOTI ou Cahiers d'Études sur la Méditerranée orientale et le monde turco-iranien. La nouvelle série, codirigée par Ali Kazancigil et Georges Prévélakis, est éditée par CNRS Éditions. Ce troisième volume, publié en septembre 2012, dirigé par Catherine Wihtol deWenden, entièrement consacré à un dossier intitulé " laTurquie, au carrefour des turbulences migratoires ", se présente en quatre parties : 1- Istanbul, un cosmopolitisme d'hier et aujourd'hui (textes d'Altan Gökalp, Luisa Piart, Jean-François Pérouse) ; 2-Échanges de population entre Grèce et Turquie et à Chypre (textes de Michel Bruneau, Meropi Anastassiadou, Nicolas Kazarian) ; 3- Anciens et nouveaux visages de l'émigration et de l'immigration dans la région (textes d'Ural Manço, Benjamin Bruce, Deniz Akagül, Farkhad Alimukhamedov) ; 4- Frontières et liens (textes d'Adeline Braux, Faruk Bilici, Fabien Dany, Gilles Riaux). La recherche d'un équilibre entre parties est parfois un peu artificielle : relatif à la politique migratoire de l'Ouzbékistan, le texte de Farkhad Alimukhamedov aurait pu tout aussi bien figurer dans la quatrième partie centrée sur les républiques de l'ancienne URSS.

Le volume alterne, par ses thèmes et contributeurs, des considérations géographiques, historiques et politiques. Après l'introduction rédigée par Catherine Wihtol de Wenden (pp. 9-16), il débute la première partie centrée sur le cas spécifique et largement débattu d'Istanbul par une reprise d'un article d'Altan Gökalp (à la mémoire duquel le volume est d'ailleurs dédié) intitulé "Turquie, tous les chemins mènent à Istanbul " (pp. 17-22), initialement paru en 1993 dans la revue Panoramiques $^{2}$. Cette reprise introduit parfaitement la première partie par ses observations pertinentes d'une agglomération redevenant ville-monde, selon l'expression de Fernand Braudel.

Luisa Piart, « Le lien entre le commerce à la valise et l'industrie de la confection à Istanbul " (pp. 23-40), termine en Allemagne une thèse sur ce thème. Avec Michel Peraldi (son co-directeur de thèse) et Alain Tarrius, toujours à l'affût des tendances nouvelles et souvent difficiles à cerner dans un premier temps, Luisa Piart s'est spécialisée dans l'étude des relations entre commerce à la valise - tchelnoki en russe, thème de plusieurs études -, économie informelle et stabilisation d'une immigration de travail elle aussi largement informelle. Elle décrit le glissement géographique des activités de confection, par exemple de Sultanhamam à Lâleli, le rôle des réseaux d'accueil des muhacirs accueillant les nouveaux venus (Turcs de Bulgarie, Azerbaïdjanais d'Iran, etc.), mais aussi les évolutions parfois très rapides de ces relations de cette " économie de bazar " comme la nomme Michel Péraldi.

Jean-François Pérouse, géographe très productif et particulièrement intéressé par l'évolution de la gigantesque agglomération d'Istanbul, renverse avec " L'afflux récent des Européens à Istanbul : inerties et recompositions des profils et des positions " (pp. 41-56) I'analyse habituelle sur la Turquie, pays d'émigration, en examinant un sujet peu connu et assez difficile, du fait de la rareté des sources sur un phénomène relativement nouveau. La Turquie, pays d'immigration, est connue pour ses flux traditionnels (muhacirs et göçmens, réfugiés rapatriés d'origines

2 Gökalp Altan (1994) En Turquie, tous les chemins mènent à Istanbul, Panoramiques, 14 , pp. 172-174. 
ottomanes et turciques), sa position de hub des migrations irrégulières (que l'auteur a largement décrit), sa nouvelle position de pays d'immigration de populations peu qualifiées (comme celle des Africains étudiée par des chercheurs de plus en plus nombreux $)^{3}$. Ici, Jean-François Pérouse expose le cas des cadres expatriés, professeurs et intellectuels (catégories en retrait et pour les seconds en voie de précarisation), artistes, retraités, mais aussitôt pour nuancer le discours par des observations fines, comme le nombre très important des Euro-Turcs, binationaux, enfants d'immigrés turcs d'origine venus profiter de taux de croissance à faire pâlir la plupart des économies de I'UE.

Dans la deuxième partie, Michel Bruneau, avec une contribution intitulée "L'expulsion et la diasporisation des Grecs d'Asie mineure et de Thrace orientale (1914-1923) " (pp. 57-84), revient sur un sujet qu'il connaît bien : les effets migratoires de la Première Guerre mondiale et de la guerre d'Indépendance et la quasi-élimination d'une population autochtone remontant au moins à 3000 ans de présence en Anatolie. L'article élargit la réflexion à toutes les composantes helléniques de la région : Istanbul et Thrace, région égéenne, Pont, Cappadoce et Chypre, montrant les effets du nationalisme de défense intransigeant qui continue à marquer la politique intérieure turque aujourd'hui encore. Si les Arméniens en ont été indéniablement les principales victimes, les Grecs d'Asie mineure ont connu des épreuves à répétition sur une période plus longue encore, pour un résultat démographiquement équivalent (éradication des

3 Comme une récente livraison de la revue de la Faculté des Sciences politịques de I'Université d'Ankara (Ankara Universitesi SBF Dergisi, 68, janvier-mars 2013) largement consacrée à la présence africaine en Turquie. minorités chrétiennes autochtones), comme le montre l'article suivant.

Méropi Anastassiadou, dans son article intitulé " D'une rive à l'autre. Les migrations des Grecs de Turquie depuis la Seconde Guerre mondiale " (pp. 85-100), traite un sujet bien moins connu, celui des expulsions ou des fuites des Grecs stambouliotes après 1945. Largement entamé dans la période précédente (travaux de Michel Bruneau), l'exil des rescapés du traité de Lausanne a continué jusqu'à la quasi-disparition de cette population autochtone de Turquie, avec de brusques à-coups, comme en 1955 ou 1964. II a continué par le biais de l'émigration de travail, intégrant discrètement nombre de réfugiés de facto (lors du coup d'État de 1970) ou de jure (autour du coup d'État de 1980). Le résultat d'ensemble est simple à énoncer : les effets du traité de Lausanne (1923) ont été petit à petit réduits à néant ou presque (Grecs, Arméniens, Chrétiens orientaux généralement dits en Turquie AssyroChaldéens). L'auteure insiste très justement sur les ambiguïtés d'un pouvoir " démocrate musulman " qui d'une part ne déroge pas aux politiques précédemment suivies en ce qui concerne le traitement des minorités non musulmanes (sous couvert de dialogue interreligieux et de tolérance), d'autre part est largement adepte du double langage propre à endormir les décideurs européens en charge du dossier d'élargissement de I'UE à la Turquie.

Nicolas Kazarian, avec un article intitulé "La question chypriote aux prises avec les enjeux migratoires de la Turquie " (pp. 101-120) fait le point sur une question lancinante, assez 
rarement traité $e^{4}$. Cet article complète les études précédentes de Michel Bruneau et Méropi Anastassiadou : I'immigration-colonisation vient ici très imparfaitement compenser l'exode des Chypriotes grecs vers le sud (que I'on pourrait qualifier aujourd'hui d'épuration ethnique) mais aussi celui des Chypriotes turcs vers la GrandeBretagne insatisfaits de la politique d'Ankara comme de celle de Nicosie. L'article décrit les résistances passives desTurcs autochtones, regroupés dans la partie nord (République turque de Chypre Nord, état non reconnu par la " communauté internationale "). L'actualité récente est cruelle pour les deux parties chypriotes, ce qui n'était pas à l'ordre du jour au moment de la rédaction de l'article : vaut-il mieux vivre dans un pays en faillite financière, " trahi " par I'UE (sud) ou sous le contrôle d'un pays basculant dans un régime totalitaire, " abandonné " par I'UE (nord) ?

La troisième partie est centrée sur l'immigration en Europe, à la notable exception de Farkhad Alimukhamedov. Dans un court article, "La dimension démographique et les caractéristiques sociales et économiques de l'émigration turque en France " (pp. 121-130), Ural Manço revient sur les caractéristiques générales de l'immigration turque : grande diversité des origines ethniques et confessionnelles, historique complexe d'une immigration souvent discrète et méconnue mais qui approche les cinq décennies de présence, évolutions contrastées qui à la fois montrent des progrès encourageants dans ce qu'il est convenu de nommer l'intégration, mais forte identité turco-musulmane d'ensemble qui ici n'est pas jugée contradictoire ou problématique, dernier point qui sera peut-être réévalué à moyen terme, à

4 Assez rarement traitée, mais cependant pas absente de la recherche francaise : il est possible de citer des écrits d'Étienne Copeaux, Gilles Bertrand, etc. la suite des événements vécus en Turquie durant l'été 2013.

Benjamin Bruce, avec " Gérer l'islam à l'étranger : entre service public et outil de la politique étrangère turque $"$ (pp. 131-148), se penche sur un sujet sensible, qui prend plus de relief encore avec l'évolution très récente (au moment de la rédaction de cette note, juillet 2013) de la politique intérieure turque (et d'ailleurs renvoie à l'article précédent en focalisant la réflexion sur ce point précis). Ce sujet est celui de l'usage du religieux pour des fins politiques n'ayant pas grand-chose à voir avec la dimension spirituelle. Celle-ci peut rapprocher, un usage identitaire et idéologique, une façon de soft-power alaturka, peut au contraire diviser. Dès les premières publications de Riva Kastoryano (années 1980), on avait compris que la dimension religieuse pour l'immigré "lambda " excédait de très loin le discours sur la Turquie laïque parce que kémaliste. II est vrai, comme le souligne l'auteur, qu'on manque souvent d'études fines sur le phénomène religieux turc en Europe, mais rappelons cependant quelques écrits de Hamit Bozarslan ou Franck Frégosi qui ont cerné assez tôt les caractéristiques de cet islam anatolien immigré appelé à remplacer les passeurs initiaux, originaires de la gauche turque. Benjamin Bruce propose une lecture actuelle de ces mouvements turcs en Europe, souvent considérés comme relevant de l'islam oppositionnel (ce qu'ils ont pu être effectivement) et qui aujourd'hui forment le ban et I'arrière-ban de la droite conservatrice (et, depuis quelques mois, ouvertement réactionnaire).

Économiste et universitaire, Deniz Akagül, dans "Dynamiques migratoires en Turquie : bref aperçu éclectique " (pp. 149-164) propose un état général de I'ensemble des flux migratoires touchant la Turquie ; la Turquie est effectivement pays de migrations internes (en particulier exode rural depuis les années 1950), pays d'émigration, pays d'immigration, pays de transit. 
Farkhad Alimukhamedov prépare une thèse sur les migrations estudiantines ouzbèkes vers les pays de I'UE. Son article, "Les particularités de l'Ouzbékistan dans la gestion de ses migrations internationales " (pp. 165-188), montre que contrairement à d'autres pays voisins confrontés aux mêmes questions (Azerbaïdjan, Arménie, Kazakhstan, Kirghizie et... Russie), I'Ouzbékistan n'a pas su, ou ne veut pas, instrumentaliser son émigration sous la forme d'une diaspora contrôlée par les pouvoirs publics ${ }^{5}$. Fierté nationale (ne pas accepter l'idée que la société dépend en grande part des revenus de l'émigration), méfiance face aux interférences politiques que génère toute forme de diaspora ? Toujours est-il que le fait migratoire est ici minimisé, considéré comme relevant du droit commun, ce qui n'empêche évidemment pas les tensions.

La quatrième partie est plutôt dédiée aux relations avec l'extérieur (ex-URSS, Iran, reste du monde par le biais des migrations irrégulières.) Adeline Braux propose un bilan pour une question peu connue en Europe occidentale : " Les migrations en provenance des ex-républiques soviétiques en Turquie, vingt ans après la disparition de I'URSS " (pp. 189-202). Elle insiste sur la stabilisation de réalités très nouvelles dans cette vaste région du monde, longtemps restée sourde aux faits sociaux que connaissait le reste de la planète : la migration est devenue très courante, la liberté de circuler est largement instaurée (accessibilité des routes internationales de migration, passage des frontières), la variété des formes de migration est extrême : toutes choses inconnues à l'époque soviétique. L'article fait un point spécifique à la Turquie, pays d'immigration et de transit, pour les ex-Soviétiques

5 Voir les textes de Thorez Julien et Massot Sophie sur l'Ouzbékistan, Rumyansev Sergei sur l'Azerbaïdjan ou Mendikulova Gulnara sur le Kazakhstan, etc. (2010) Revue Européenne des Migrations Internationales, 26 (3), 233 p comme pour les Africains ou les Moyenorientaux.

Dans I'article « Sarp/Sarpi : la porte de l'intégration entre la Turquie et la Géorgie vingt ans après la chute de I'Union soviétique " (pp. 203-222), Faruk Bilici revient sur une étude réalisée juste après la dislocation de I'URSS et publiée dans Panoramiques et les CEMOTF.A l'époque, la Turquie avait pris la décision d'ouvrir deux "portes" vers la Géorgie, Sarp et Türkgözü, et une vers I'Azerbaïdjan, ou plutôt l'enclave azerbaïdjanaise entre Arménie, Turquie et Iran, le Nakhitchevan (pont et douane de Dilucu/Sederek) permettant alors à ce petit territoire d'échapper au conflit meurtrier du Haut Karabagh. Le bilan est d'autant plus intéressant que l'auteur connaît bien ce site ; alliant observations de terrain très précises, utilisation des statistiques et observations politologiques, Faruk Bilici fait véritablement ici œuvre de géographe (avec quelques photos de l'auteur bien choisies pour illustrer le propos). L'article montre bien les avancées en dents de scie, déclarations d'intention insistant sur l'ouverture, vite contrecarrées par la réalité quotidienne, mais rattrapées et dépassées par cette même réalité, économique et sociale, qui malgré tout - et contre l'avis de décideurs qui ne sont pas sur place - finit par s'imposer. À Sarp et autour (département turc d'Artvin, république autonome géorgienne d'Adjarie), se mettent en place des coopérations transfrontalières, des relations régionales (à l'échelle du Caucase) et internationales (avec I'Asie centrale), " renaissance effective de la route de la Soie ? ", comme s'interroge l'auteur.

Fabien Dany aurait pu figurer dans la première partie. Avec " La Turquie, nouveau hub des migrations clandestines vers l'Europe ? " (pp. 223-234), il revient

6 Faruk Bilici (1994) À la frontière turcogéorgienne..., Panoramiques, 14, pp. 167-171 ; Faruk Bilici (1993) Une réalisation concrète avant la lettre : la coopération en Mer noire orientale, CEMOTI, 15, pp. 169-183. 
sur l'actualité d'une question sensible dans les relations turco-européennes, celle du pont migratoire que forme I'Anatolie entre Balkans, Afrique orientale, Moyen-Orient et Caucase. La Turquie joue ici un rôle ambigu, associée à l'Europe dans la lutte contre la migration irrégulière (comme sa collaboration avec l'agence Frontex ou l'ouverture de centres de rétention), mais selon des critères qui lui sont souvent propres (restriction du droit d'asile et gestion pour le moins opaque de ce droit, politique spécifique des visas, etc.) comme si les migrations irrégulières servaient de variable d'ajustement dans la négociation avec I'UE.

Pour le dernier article de ce numéro, “ Par-delà le Caucase et l'Anatolie. La genèse transnationale de la cause azerbaïdjanaise en Iran " (pp. 235-258), Gilles
Riaux, spécialiste de la recherche identitaire en Azerbaïdjan iranien, montre comment jouent des facteurs contradictoires dans un triangle déjà ancien : Turquie, République islamique d'Iran et Fédération de Russie prennent la suite des trois empires (ottoman, persan et russe) pour une population autochtone turcophone divisée par des frontières étatiques assez stables, mais plus par des constructions politiques très antagonistes. Les relations perdurent, se réadaptent en tant que besoin, malgré les assignations des administrations centrales, dessinant de facto un grand Azerbaïdjan transfrontalier qui ne peut laisser les gouvernements centraux indifférents et à tout moment raidir les relations entre Téhéran, Bakou et Ankara.

Stéphane de Tapia CNRS/Université de Strasbourg 